\title{
Avoidance of a warning signal as a function of the signal termination contingency*
}

\author{
PAUL LEWIS \\ Ohio University, Athens, Ohio 45701 \\ and \\ PIETRO BADIA \\ Bowling Green State University, Bowling Green, Ohio 43403
}

Four rats were conditioned to avoid shock on a fixed-cycle (15-sec) avoidance schedule. A 6-sec tone was introduced preceding shock. The tone could be avoided by a single response within the cycle preceding the tone's onset. None of the four rats consistently avoided the tone, and this effect did not depend upon whether the tone could be terminated by the $S$. These results suggest that a warning signal does not function as an aversive stimulus, if the aversiveness of a stimulus is defined in terms of maintaining avoidance behavior. Finally, possible reasons for the inconsistency between the present experiment and that of Logan \& Boice (1968) are noted.

Experimenters have observed that, if a warning stimulus is provided just preceding shock on a free-operant avoidance schedule, animals wait for the stimulus and respond in its presence (Sidman, 1955). If, however, the contingency is changed so that once the signal is permitted to come on it cannot be terminated and shock can no longer be avoided, then Ss tend to avoid both the signal and the shock (Sidman \& Boren, 1957). The latter procedure (Sidman \& Boren, 1957) differs from the former (Sidman, 1955) in that the shock avoidance contingency and the signal termination contingency both changed. Either of these two variables could account for the dissimilar behavior patterns. The present investigation held the shock avoidance requirement constant and varied the signal termination contingency.

\section{METHOD}

\section{Subjects}

Four naive female albino rats were used (Holtzman Company). Ss were about 100 days old at the start of training and were maintained in individual cages on a 12-h light-dark cycle, with free access to food and water.

\section{Apparatus}

An operant chamber 10.5 in. long and $10 \mathrm{in}$. wide, with a sloping Plexiglas roof, was used as the experimental space. The roof attached to the 10 -in. wall approximately 5 in. above the grid floor. Two response levers, requiring approximately $20 \mathrm{~g}$ pressure to operate microswitches, were present in the chamber. Only the right lever, however, was involved in this experiment. Levers were centered 2 in. from the side along the 10-in. wall. A

*A paper based on these data was presented at the Southeastern Psychological Association meeting, Miami, 1971. Reprints may be obtained from Paul Lewis, Department of Psychology, Porter Hall, Ohio University, Athens, Ohio 45701. stimulus light was located above the clear Plexiglas roof and a $1400-\mathrm{Hz}$ tone (approximately $86 \mathrm{~dB}$ ) served as the warning signal. The entire chamber was enclosed in a ventilated metal shell (83-dB background level).

A constant-wattage shock source (BRS, Inc.) delivered .32-sec shocks at $75 \mathrm{~mW}$. The walls and response lever featured as one contact in the grid scrambling circuit, which reversed polarity between each contact 50 times per second. Data were recorded automatically on counters and a cumulative recorder.

\section{Procedure}

Three avoidance schedules were used. The fixed-cycle schedule introduced by Sidman $(1962,1966)$ was the basic procedure. Two signaled avoidance schedules, which were variations of the fixed-cycle schedule, were compared with it. Fixed-cycle avoidance (no-signal condition) involved delivering a brief electric shock at fixed intervals $(15 \mathrm{sec})$ until a response occurred. A single response omitted shock scheduled to occur at the end of the cycle. Responses, of course, could occur at any point during the cycle, but only the first response in each cycle affected shock. In addition to the unsignaled fixed-cycle condition, a signal terminate condition and a signal no-terminate condition were studied.

The signal terminate schedule was identical to the no-signal procedure, except that each shock was preceded by a 6 -sec tone. On this schedule, also, each response during a cycle omitted shock but, in addition, each response preceding the tone omitted both tone and shock. A response during the tone terminated it and omitted shock.

The signal no-terminate schedule was identical to signal terminate except for the response contingency during the tone. On the signal no-terminate schedule, a response during tone omitted shock but did not terminate the signal.

\section{RESULTS}

The number and sequence of conditions are given in Table 1. Two Ss received the signaled schedules in the order signal terminate followed by signal no-terminate; two Ss received the schedules in the reverse order. A given schedule remained in effect until rate and pattern of responding were reasonably constant over a minimum of three sessions (as determined by inspection of the cumulative records). A stimulus light above the Plexiglas roof was on before and after, but not during, each 6-h session. Sessions were conducted every other day. For response feedback, each leverpress provided power to the cue light over the lever for $75 \mathrm{msec}$.

All four Ss learned to avoid shock efficiently. The mean range in the percentage of shocks received during the final nine asymptotic sessions was 1.8-5.2 for S 218 , 0.6-16.6 for S 3, 6.5-27.2 for S 2, and 3.6-14.7 for $\mathrm{S} 215$. Shock rate did not show a consistent relationship to the three avoidance schedules.

Primary interest in this experiment centered around whether or not Ss avoided the warning signal. Figure 1 shows the number of warning signals received by each $S$ during the final three sessions on each schedule. The maximum number of signals possible during a single session was 1,440 . On the signaled fixed-cycle schedules, 
Table 1

Number of Sessions Required to Stabilize Avoidance Performance on Each Schedule for All Ss in the Experiment

\begin{tabular}{clr}
\hline S Number & \multicolumn{1}{c}{ Schedule } & Sessions \\
\hline \multirow{2}{*}{218} & No Signal & 5 \\
& Signal No-Terminate & 11 \\
& Signal Terminate & 8 \\
& No Signal & 3 \\
& No Signal & 8 \\
& Signal No-Terminate & 10 \\
& Signal Terminate & 16 \\
& No Signal & 3 \\
& No Signal & 5 \\
& Signal No-Terminate & 12 \\
& Signal Terminate & 7 \\
& No Signal & 6 \\
& No Signal & 9 \\
& Signal Terminate & 6 \\
& Signal No-Terminate & 17 \\
& Signal Terminate & 9 \\
& No Signal & 3 \\
\hline
\end{tabular}

Ss could respond prior to the signal and thus avoid the signal and shock or they could "wait" for the signal and respond in its presence.

Figure 1 shows that none of the four Ss avoided the warning signal under signal terminate or signal no-terminate schedules when compared to the no-signal condition. Ss 218 and 3 received many more warning signals (center two panels) under the no-terminate and terminate signal schedules than under the no-signal schedule. (Of course, the warning signal never occurred on the no-signal schedule. This simply refers to the number of times the behavior would have permitted the signal to occur if it were enabled.) The same trend appears in the data for S 215 but is not as marked. S 2 received more signals under the signal terminate condition than under the no-terminate or no-signal
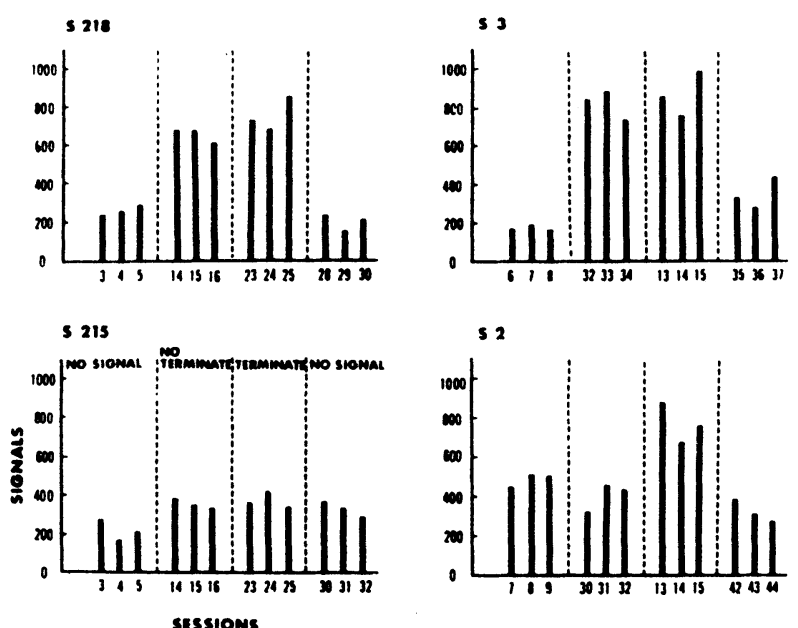

Fig. 1. Shows total warning signals received during final asymptotic sessions on each schedule for all Ss in the experiment. condition, but the latter two schedules did not differ.

While Ss showed a similar tendency not to avoid the warning stimulus under both terminate and no-terminate conditions, the termination contingency was not without effect. S 2 avoided more signals on the no-terminate schedule than on the terminate and Ss 215 , 218 , and 3 showed slight trends in the same direction (see Fig. 1). In addition, all Ss required somewhat longer to stabilize on the no-terminate schedule than on the terminate (see Table 1).

\section{DISCUSSION}

The free-operant avoidance schedule places an advantage on responses occurring near the end of the response-shock interval. A preshock warning signal may produce spaced responding simply because it accentuates this advantage for spaced responding. Previous research has demonstrated that Ss wait for a preshock stimulus on the adjusting avoidance schedule (Field \& Boren, 1963), and the present experiment shows the same effect on the fixed-cycle schedule. These findings indicate that Ss will wait for and respond in the presence of a preshock stimulus, even when there is no temporal advantage in doing so.

The tendency to wait for the warning signal and respond in its presence on the fixed-cycle schedules is consistent with the pattern of responding observed on the signaled free-operant avoidance schedule (Sidman, 1955). In Sidman's and other previous experiments, the warning signal could typically be terminated by a response in its presence. The no-terminate condition in the present experiment eliminated that contingency and Ss still failed to avoid the warning signal. These data indicate that lack of aversiveness of the warning signal is not due to Ss terminating the stimulus shortly after it comes on.

Ss received slightly fewer signals during the no-terminate condition than during the terminate condition, although they did not consistently avoid the signal under either. This could be due to response feedback not being as effective on the no-terminate schedule as it was on the terminate schedule. While all responses were followed by approximately $75 \mathrm{msec}$ of illumination, offset of the signal provided additional stimulus change (feedback) on the terminate schedule. The significance of the feedback variable in facilitating the acquisition of avoidance behavior was demonstrated by Bolles \& Popp (1964). Perhaps feedback also improves steady state performance.

Recently, Logan \& Boice (1968) reported data on a signaled and unsignaled fixed-cycle schedule similar to the ones used here. The cycle they used was $20 \mathrm{sec}$ in duration, ending in a shock which had to be terminated (escape) by a response. The warning signal was a 4-sec light which terminated with a bar response. Logan and Boice presented their data in terms of the frequency distribution of responses over the 20 -sec cycle. The data and conclusions of Logan and Boice were the opposite of those presented here. In their experiment, Ss tended to respond early in the cycle and, hence, avoid the warning signal. Logan and Boice concluded that avoidance of the warning signal was reinforcing.

While a number of parameters and procedures differ between the present study and the Logan and Boice experiment, one factor stands out as the one most likely to account for the different results. Rats in the Logan and Boice study probably did not reach a steady state performance level and were in a shock-elicited or pseudoavoidance pattern of responding. The 50\%-60\% shock avoidance levels reported by Logan and Boice are consistent with this suggestion. On a fixed-cycle schedule, shock-elicited responding results in avoiding every other shock or approximately $50 \%$ avoidance.

Although postshock responses on the signaled fixed-cycle schedule have the effect of avoiding the warning signal, these responses are probably the result of variables other than the signal avoidance contingency. Hence, Logan and Boice's 
conclusion that avoidance of the warning signal reinforces responding does not seem justified. Ss in the present study avoided approximately $90 \%$ of the scheduled shocks and could not have been responding in the postshock pattern. When pseudoavoidance was minimized (presumably by the longer sessions or one of the temporal parameters), no evidence for negative reinforcement from warning signal avoidance was observed.

\section{REFERENCES}

Bolles, R. C., \& Popp, R. J., Jr. Parameters affecting the acquisition of Sidman avoidance. Journal of the Experimental Analysis of Behavior, 1964, 7, 315-321.

Field, G. E., \& Boren, J. J. An adjusting avoidance procedure with multiple auditory and visual warning stimuli. Journal of the Experimental Analysis of Behavior, 1963, 4, 537-543.

Logan, F. A., \& Boice, R. Avoidance of a warning signal. Psychonomic Science, 1968, 13, 53.

Sidman, M. Some properties of the warning stimulus in avoidance behavior. Journal of Comparative \& Physiological Psychology, 1955, 48, 444-450.

Sidman, M. Classical avoidance without a warning stimulus. Journal of the Experimental Analysis of Behavior, 1962, 5 , 97-104.

Sidman, M. Avoidance behavior. In W. K. Honig (Ed)., Operant behavior: Areas of research and application. New York: Appleton-Century-Crofts, 1966.

Sidman, M., \& Boren, J. J. A comparison of two types of warning stimulus in an avoidance situation. Journal of Comparative \& Physiological Psychology, 1957, 50, 282-287.

(Received for publication January 29, 1973.) 